\title{
Bioequivalence of Two Formulations (Innovator vs Generic) of Capecitabine in Patients with Cancer of Colon
}

\author{
Ruiz-García Erika Betzabe ${ }^{1}$, Mancera-Reyes Víctor Manuel ${ }^{2}$, Bustillos-Ventura Rafael ${ }^{2}$, \\ Cárdenas José Manuel $^{2}$, Canales-Vázquez Emmanuel ${ }^{3}$, Meixueiro-Montes de Oca Raúl ${ }^{3}$ \\ ${ }^{1}$ Nanopharmacia Diagnóstica, Mexico City, Mexico \\ ${ }^{2}$ Research, Science and International Technology, Mexico City, Mexico \\ ${ }^{3}$ Clinical Research Department, Laboratorios PiSA, S.A. de C.V, Mexico City, Mexico
}

\section{Email address:}

jmeixueiro@pisa.com.mx (Meixueiro-Montes de O. R.), betzabe100@yahoo.com.mx (Ruiz-García E. B.), vmancera@ictinternacional.com (Mancera-Reyes V. M.), rbustillos@ictinternacional.com (Bustillos-Ventura R.), ictcardenas@aol.com (C. J. Manuel), ecanales@pisa.com.mx (Canales-Vázquez E.)

\section{To cite this article:}

Ruiz-García Erika Betzabe, Mancera-Reyes Víctor Manuel, Bustillos-Ventura Rafael, Cárdenas José Manuel, Canales-Vázquez Emmanuel, Meixueiro-Montes de Oca Raúl. Bioequivalence of Two Formulations (Innovator vs Generic) of Capecitabine in Patients with Cancer of Colon. Cancer Research Journal. Vol. 3, No. 6, 2015, pp. 110-114. doi: 10.11648/j.crj.20150306.11

\begin{abstract}
Capecitabine is an orally administered chemotherapeutic agent used in the treatment of numerous cancers including colon, colorectal, ovarian, breast and pancreatic. Considering the importance of generic drugs in Health Care Systems, it is essential that its quality, safety and efficacy be compared with the corresponding innovator product. The objective of the study was to compare the pharmacokinetics and relative bioequivalence between two tablet (500 mg) formulations of capecitabine in Mexican patients with cancer of colon. The study was designed as open, prospective, randomized, two-way, crossover bioequivalence trial. A single oral dose of $2000 \mathrm{mg}$ capecitabine was administered on two separate days to 24 patients. After each administration, serial blood samples were collected for up $8 \mathrm{hr}$. The washout between the two administrations was 3 days. Capecitabine was determined in plasma using LC/MS-MS. No statistically significant differences in $\mathrm{C}_{\max }, \mathrm{AUC}_{0-\mathrm{t}}$, and $\mathrm{AUC}_{0-\alpha}$ were found between the test and innovator formulations. Both products were well tolerated by the patients, with no serious adverse events. The generic capecitabine was pharmacokinetic bioequivalent with the innovator formulations.
\end{abstract}

Keywords: Capecitabine, Generic, Pharmacokinetic, Bioequivalence, Cancer, Colon

\section{Introduction}

Capecitabine a fluoropyrimidene carbamate rationally designed as orally administered is currently approved by the FDA (Food and Drug Administration) EMA (European Medicinal Agency) and COFEPRIS (Federal Commission against Sanitary Risks, Mexico) as adjuvant in patients with colon, colorectal [1-3], breast [4, 5], ovarian [6, 7] and pancreatic [8-10] cancer, in combination with other antineoplasic drugs. Capecitabine a prodrug is selectively activated by tumor cells to its cytotoxic moiety, 5-fluorouracil (5-FU), by thymidine phosphorylase, which is generally expressed at high levels in tumors [11, 12]. Following oral administration, capecitabine is rapidly and almost completely absorbed as an intact molecule, and undergoes a three-step enzymatic conversion to 5-FU [13].
Capecitabine and its intermediate metabolites, 5-Deoxy-5-Fluorouracil (5-DFUR) are not intrinsically cytotoxic. In the final step, the intermediate 5-DFUR is converted to 5-FU by the enzyme thymidine phosphorylase, which has significantly higher activity in tumor than the normal tissue [13-16].

Capecitabine has almost $100 \%$ oral bioavailability and exhibits linear increases in maximum plasma concentration $\left(\mathrm{C}_{\max }\right)$ and area under curve (AUC) with dosage increases [13]. After two doses of $1250 \mathrm{mg} / \mathrm{m}^{2}$, the drug undergoes rapid absorption, with peak plasma levels of $3.9 \mathrm{mg} / \mathrm{L}$ in 1.5 to 2.0 hours. In comparison $\mathrm{C}_{\max }$ for the active metabolite, 5-FU is lower at $0.66 \mathrm{mg} / \mathrm{L}$ with similar time to reach maximum concentration $\left(\mathrm{T}_{\max }\right)$ of 2 hours. AUC values for the parent drug and active metabolite for the same dosage are 5.96 $\mathrm{mg} * \mathrm{~h} / \mathrm{L}$ and $1.34 \mathrm{mg} * \mathrm{~h} / \mathrm{L}$, respectively [13]. The elimination 
half-life $\left(t_{1 / 2}\right)$ of capecitabine is short, ranging from 0.49 to 0.89 hours. The $t_{1 / 2}$, for the 5 -FU metabolite is slightly longer at 0.67 to 1.15 hours $[14,17,18]$. Recovery of drug related material in urine and feces is nearly $100 \%$ [12].

The recommended single-agent dose of capecitabine is 1250 $\mathrm{mg} / \mathrm{m}^{2}$ twice daily (at 12 -h interval), days 1 to 14 followed by a 7-day rest period. In patients with colon cancer, the standard single-agent dose of capecitabine is well tolerated, with a low incidence of grade $3 / 4$ adverse events and a particularly low incidence of myelosuppression [19]. The incidence of adverse events observed in breast and colon cancer patients after 2500 $\mathrm{mg} / \mathrm{m}^{2}$ per day was as follows: $1-10 \%$ taste disturbance, chest pain, alopecia, gastrointestinal hemorrhage, thrombocytopenia, cough, venous thrombosis, arthralgia/myalgia and headache; $11-20 \%$ dyspnea, paresthesia, eye irritation, edema, constipation, and neutropenia $[11,20]$.

The high antitumor activity and favorable safety profile, particularly the low rate of myelosuppression of capecitabine, make it an attractive agent alone or for incorporation into regimens.

In Latin America, the limited access for high-quality drugs, specifically anti-neoplasic drugs, and due to cost of treatment constitutes an unmet medical need in these countries. The use of generic drugs has already decreased the cost of treatment in many regions of the world. The approval of generics by regulatory agencies is critical to facilitate patient access to this kind of drugs and will allow governments to afford the cost of these pathologies.

The key to generics development is the demonstration of similarity since the pharmacokinetic, pharmacodynamics safety and efficacy point of view to achieve commercial viability, represented by a broad product label. The aim of this study was to evaluate the pharmacokinetic of two-capecitabine tablets formulation, innovator versus generic in patients with colon cancer.

\section{Subjects and Methods}

\subsection{Study Design and Study Population}

The trial was a comparative, prospective, crossover, open-label pharmacokinetic study performed in 24 Mexican patients with colon cancer diagnostic. The trial was conducted in full agreement with Helsinki declaration and by the COFEPRIS (Mexican Regulatory Agency). The Ethical Committee in Research of Investigación, Ciencia y Tecnología Internacional (ICT), approved the trial protocol. In addition, each patient, following evaluation of the oncologist, provided written informed consent. Screening included physical examination, medical history, vital signs, laboratory tests (hematology, blood chemistry, and urinalysis) tumor assessment, electrocardiogram (ECG) and for females pregnancy test. In addition, safety parameters (adverse events, laboratory tests, and vital signs) were assessed on treatment days and at follow up.

\subsection{Inclusion/Exclusion Criteria}

The eligibility criteria and clinical assessments were: man or women, age 18-70 years old, histologically/cytologically confirmed colon cancer, Karnofsky between 70 and 100\%, a life expectancy of at least 12 weeks, adequate hematopoietic function; adequate hepatic function including total bilirubin levels less than 1.5 times the upper limit of normal (UNL), and aspartate transaminase (AST), alanine transaminase (ALT) and alkaline phosphatase $<2.5$ times UNL; and adequate renal function including serum creatinine $<1.5$ times UNL. The exclusion criteria were cardiac, hepatic, renal, pulmonary, neurological, and hematological diseases, pregnancy, alcoholism, drug abuse, hypersensitivity to capecitabine, had participated in any other clinical study in the last 3 months and any other acute or chronic condition that can alter the results interpretation.

\subsection{Clinical Procedure}

The patients were randomly assigned to the treatment sequences $\mathrm{A} / \mathrm{B}$ or $\mathrm{B} / \mathrm{A}$. The patients had a standard breakfast $30 \mathrm{~min}$ before the capecitabine administration. The day of the study received a single dose of $2000 \mathrm{mg}$ capecitabine as four tablets formulation A (reference treatment; Xeloda, Roche) or a single dose of $2000 \mathrm{mg}$ capecitabine as four tablets of formulation B (test treatment; Laboratorios PiSA). The washout period between the two treatments was 3 days.

\subsection{Sampling Technique}

For pharmacokinetic assessment, $5 \mathrm{~mL}$ blood samples from a suitable antecubital vein were collected in vacutainers containing EDTA as anticoagulant at the following time-points: predose $0.0,0.167,0.333,0.500,0.667,0.833$, $1.000,1.167,1.333,1.500,1.667,1.833,2.000,2.500,3.000$, 6.000 and 8.000 hours after capecitabine intake. Blood samples were immediately centrifuged $(10 \mathrm{~min}$ at $3,000 \mathrm{~g}$, room temperature). The supernatant plasma was removed and stored in plastic tubes at $-70^{\circ} \mathrm{C}$ until analysis. Plasma concentrations of capecitabine were determined by a validated liquid chromatography with mass-spectrometry detection (LC/MS-MS) [13].

\subsection{Pharmacokinetic Parameters Evaluated}

Pharmacokinetic parameters were assessed by standard non-compartmental analysis using Win Non Lin version 6.3. The pharmacokinetic parameters of capecitabine were estimated for each patient from the concentration-time data on study days 1 and 4 . Maximum plasma concentration $\left(\mathrm{C}_{\max }\right)$ and the time to reach this value $\left(\mathrm{t}_{\max }\right)$ were determined from the highest observed concentration and the time, which it occurred. Apparent $t_{1 / 2}$, was estimated from $\ln 2 / \lambda$, where the apparent rate constant elimination $\lambda$, was estimated by linear regression on the logarithm of the plasma concentration versus time data.

The area under the plasma concentration time curve from time 0 to infinity $(\mathrm{AUC} 0-\alpha)$ was estimated from the sum of 
$\mathrm{AUC}_{0-\mathrm{t}}$ and Ctlast/ $\lambda$, where $\mathrm{AUC}_{0-\mathrm{t}}$ is the area under the curve from time 0 to the last sampling time (tlast) at which a concentration above the limit of quantification was measured $\left(\mathrm{C}_{\max }\right)$. $\mathrm{AUC}_{0-\mathrm{t}}$ was calculated using the linear trapezoid rule.

\section{Results}

The study included 11 female and 13 male patients with colon cancer (age $47.63 \pm 11.8$ years, weight $63.89 \pm 10.94 \mathrm{~kg}$, height $1.59 \pm 0.093 \mathrm{~m}$, BMI $25.20 \pm 2.72 \mathrm{~kg} / \mathrm{m}^{2}$ ).
The drugs were well tolerated by the patients, and the side events were diarrhea (one patient), nausea (two patients) for generic drug and for reference drug, pain in perianal region (one patient), and abdominal pain (one patient). The biochemical and hematological parameters presented no clinically relevant alterations.

The results of the pharmacokinetic parameters $\left(\mathrm{C}_{\max }, \mathrm{T}_{\max }\right.$, $t_{1 / 2}$ and AUC), after single oral administration of $2000 \mathrm{mg}$ capecitabine in the form of tablet " $\mathrm{A}$ " and tablet " $\mathrm{B}$ " are shown in the table 1.

Table 1. Pharmacokinetic parameters (mean $\pm S D$ ) of capecitabine, after administration of generic and innovator drugs.

\begin{tabular}{llll}
\hline Pharmacokine-tic parameter & B Generic Capecitabine & A Innovator Capecitabine & T/R Relation \\
\hline $\mathrm{C}_{\max }(\mathrm{ng} / \mathrm{mL})$ & $7328.978 \pm 6327.023$ & $7391.265 \pm 5813.591$ \\
$\mathrm{AUC}_{0-\mathrm{t}}(\mathrm{ng} \mathrm{x} \mathrm{h} / \mathrm{mL})$ & $6921.009 \pm 5515.964$ & $7476.169 \pm 7056.413$ \\
$\mathrm{AUC}_{0-\alpha}(\mathrm{ng} \mathrm{x} \mathrm{h} / \mathrm{mL})$ & $8700.075 \pm 5703.990$ & $8689.393 \pm 5211.088$ \\
$\mathrm{t}_{1 / 2}(\mathrm{~h})$ & $0.795 \pm 0.867$ & 0.743 & $0.957 \pm 0.304$ \\
$\mathrm{~T}_{\max }(\mathrm{h})$ & $1.729 \pm 0.788$ & $1.687 \pm 0.760$ & $1.318 \pm 1.127$ \\
\hline
\end{tabular}

The mean peak plasma concentrations of the capecitabine biogeneric were similar to the peak of formulation A. The time to reach these peak concentrations was identical for both formulations (Fig. 1). Plasma concentrations then declined exponentially with half-lives of $0.789 \pm 0.760 \mathrm{~h}$ and $0.795 \pm$ $0.867 \mathrm{~h}$ for formulations $\mathrm{A}$ and $\mathrm{B}$, respectively.

The $\mathrm{AUC}_{0-\alpha}$ was similar after treatment with both formulations and reached $8689.393 \pm 5211.088 \mathrm{ng} * \mathrm{~h} / \mathrm{mL}$ for formulation $\mathrm{A}$ and $8700.075 \pm 5703.990$ for formulation $\mathrm{B}$. The pharmacokinetic parameters $\mathrm{C}_{\max }, \mathrm{T}_{\max }, \mathrm{AUC}_{0-\alpha}, \mathrm{AUC}_{0-\mathrm{t}}$ and $t_{1} / 2$ were comparable following administration of formulation A or B (Table 1). There was, however, a trend for slightly higher $\mathrm{AUC}_{0-\mathrm{t}}$ values after treatment with formulation B.

The primary pharmacokinetic parameter $\mathrm{AUC}_{0-\alpha}$ of capecitabine was similar between the two groups with $90 \%$ confidence interval of $0.957 \pm 0.304$. Since the $90 \%$, confidence interval was within the predefined limits of $0.80-1.20$, equivalent between the two groups was concluded for $\mathrm{AUC}_{0-\alpha}$ of capecitabine.

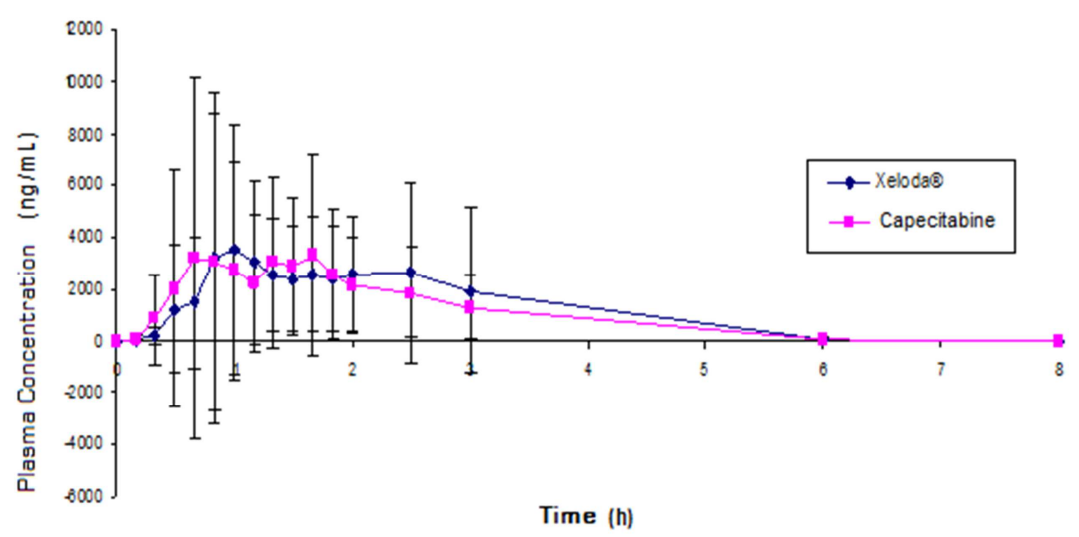

Figure 1. Graphic of the mean plasma concentration of Capecitabine vs time after administration of the two drugs, taking into account all the volunteers ( \pm S.D.).

On both study days was seen a high interpatient variability in the $\mathrm{C}_{\max }$ of capecitabine in both subgroups of patients. In addition, low intrapatients variability in the capecitabine AUC was present. As there were no major differences observed on the study of the pharmacokinetic parameters for capecitabine $\left(\mathrm{AUC}_{0-\alpha}, \mathrm{C}_{\max }\right.$ and $\left.\mathrm{t}_{1 / 2}\right)$, in the groups $\mathrm{A}$ and $\mathrm{B}$, equivalence was concluded for AUC and $t_{1 / 2}$.

\section{Discussion}

Given the current financial climate there is an ever increasing need to substitute drug treatments to optimize expenditure The need to manage and minimize costs is increasingly important for healthcare systems across the world. Generic substitution is already used widely through Europe, United States, Canada and Latin America and payers are increasingly looking towards therapeutic substitution to make additional savings [21]. These are valid methods for containing costs, particularly for conditions such as oncologic disease, where large numbers of people need to be treated in the best way to minimize disease burden. Medicines Agencies guidelines lay down the general requirements for demonstration of the similar nature of two medicinal products in terms of safety and efficacy. In this case the similar drug could be consider bioequivalent if had demonstrated efficacy and safety through clinical or bioequivalence studies. 
Demonstration of comparability between similar drugs presents a challenge.

The primary objective of this study was to compare the pharmacokinetic of two capecitabine formulations (innovator vs generic) in Mexican patients with cancer of colon. Since the composition and dissolution profiles of the two tablets were very similar, bioequivalence between the two formulations was the expected outcome of this study.

Our results show that both capecitabines were relatively rapidly and extensively absorbed following oral administration. The plasma $t_{\max }$ for the capecitabines were about $1 \mathrm{hr}$ (range 0.5 to $2 \mathrm{hr}$ ), the peak plasma drug concentration $\left(\mathrm{C}_{\max }\right)$ were of 2,500 to $3,000 \mathrm{ng} / \mathrm{mL}$ and have a relatively short elimination half-life. These results are within the conventional bioequivalence range of $80-125 \%[22,23]$

In this study, the pharmacokinetic parameters of both capecitabine was found to be similar to those found in previous studies [24-26]. The primary pharmacokinetic parameter in the present study was the $\mathrm{AUC}_{0-\alpha}$ of both drugs. The $\mathrm{AUC}_{0-\alpha}$ was chosen because a previous study [27] has shown a positive correlation between $\mathrm{AUC}_{0-\alpha}$ and efficacy and safety. The primary pharmacokinetic parameter, the $\mathrm{AUC}_{0-\alpha}$ was equivalent between the generic and innovator.

The extent and rate at which both capecitabines reached the systemic circulation appeared to be the same following treatment with formulation B compared with formulation A and equivalence could be concluded. Indeed, the $\mathrm{AUC}_{0-\alpha}$ of capecitabine following ingestion of formulation $\mathrm{B}$ was $65.6 \%$ of that following ingestion of formulation $\mathrm{A}$ and the confidence interval was $90 \%$.

The generic capecitabine pharmacokinetic profile observed in our study was very similar to innovator capecitabine.

A high interpatient variability in the systemic exposure to capecitabine was observed. Despite the high interpatient variability, no differences in capecitabine AUC was detected between the two groups.

Oral administration, along with the extensive metabolism of capecitabine and most of its metabolites contributes to high interpatient variability [27]. Similar variability in the pharmacokinetics of capecitabine has been observed in other clinical pharmacokinetic studies [27] and the results from present study are therefore consistent with previous findings.

The European Union has led the way in the regulation of similars, responding to the patent and data protection expiry of several innovator medicines in recent years. Previous authors have reviewed the manufacturing and approval process for generics, speculating on what issues might arise once such agents are introduced [28, 29].

Now in Mexico the COFEPRIS (Regulatory Agency in Health) has established the regulatory framework for assessing generics or biosimilar. In this case, the product Capecitabine, Xeloda (Roche) was the innovator product, which was already authorized in Mexico by COFEPRIS, with a similar active substance than Laboratorios PiSA. The pharmaceutical form, strength and route of administration of PiSA's capecitabine are the same as that of the reference product.
The comparability must be demonstrated in terms of quality, efficacy and safety. The comparability of efficacy is assessed via non-clinical comparative in vitro and in vivo studies, followed by clinical efficacy studies [29]. In this study, the comparable efficacy was evaluated through the pharmacokinetic study. COFEPRIS, in certain cases, as for capecitabine pharmacokinetic studies alone are suffice for obtain authorization for marketing.

\section{Conclusion}

It can be concluded from the present study, that the generic product capecitabine $500 \mathrm{mg}$ is bioequivalent to the innovator product.

\section{Acknowledgements}

Laboratorios PISA S.A. de C.V. funded the study. The sponsor review the manuscript, but the final decision on content was with the main investigators.

\section{References}

[1] Braun AH, Achterrath W, Wilke $\mathrm{H}$, et al. New systemic frontline treatment for metastatic colorectal carcinoma. Cancer 2004, 100: pp 1558-1577.

[2] Cassidy J, Tabernero J, Twelves C, et al. XELOX (capecitabine plus oxaliplatin): Active first-line therapy for patients with metastatic colorectal cancer. J Clin Oncol 2004, 22: pp 2084-2091.

[3] Zeuli M, Nardoni C, Pino MS, et al. Phase II study of capecitabine and oxaliplatin as first-line treatment in advanced colorectal cancer. Ann Oncol 2003, 14: pp 1378-1382.

[4] Gradishar WJ, Meza LA, Amin B, et al. Capecitabine plus paclitaxel as front-line: Combination therapy for metastatic breast cancer: A multicancer phase II study. J Clin Oncol 2004, 22: pp 2321-2327.

[5] O’Shaughnessy JA, Blum J, Moiseyenko V, et al. Randomized, open-label, phase II trial of oral capectibine (Xeloda) vs a reference arm intravenous CMF (cyclophosphamide, methotrexate and 5-fluorouracil) as first-line therapy for advanced/metastatic breast cancer. Ann Oncol 2001, 12: pp $1247-1254$.

[6] Vasey PA, McMahon L, Paul J, et al. A phase II trial of capecitabine (Xeloda) in recurrent ovarian cancer. Br J Cancer 2003, 89: pp 1843-1848.

[7] Rischin D, Phullips KA, Friedlander M, et al. A phase II trial of capecitabine in heavily pre-treated platinum resistant ovarian cancer. Gynecol Oncol 2004, 93: pp 417-421.

[8] Hess V, Salzberg M, Borner M, et al. Combining capecitabine and gemcitabine in patients with advanced pancreatic carcinoma: A phase I/II trial. J Clin Oncol 2003, 21: pp 66-68.

[9] Scheithauer W, Schull B, Urlich-Pur H, et al. Biweekly high-dose gemcitabine alone or in combination with capecitabine in patients with metastatic pancreatic adenocarcinoma: A randomized phase II trial. Ann Oncol 2003, 14: pp 97-104. 
[10] Stathopoulus GP, Syrigos K, Poylzos A, et al. Front-line treatment of inoperable or metastatic pancreatic cancer with gemcitabine and capecitabine: An intergroup, multicenter, phase II study. Ann Oncol 2004, 15: pp 224-229.

[11] Walko CM, Lindley C. Capecitabine a review. Clin Ther 2005, 27: pp 23-44.

[12] Krishnaumrthi SS, Brell JM, Hoppel C, et al. Phase I clinical and pharmacokinetic study of oxaliplatin, irinotecan and capecitabine. Cancer Chemother Pharmacol 2009, 63: pp 441-450.

[13] Reigner B, Blesch K, Weidekamm E. Clinical pharmacokinetics of capecitabine. Clin Pharmacokinet 2001, 40: pp 85-104.

[14] Miwa M, Ura M, Nishida M, et al Design of a novel oral fluoropyrimidine carbamate, capecitabine, which generates 5 -fluorouracil selectively in tumors by enzymes concentrated in human liver and cancer tissue. Eur J Cancer 1998, 34: pp $1274-1281$.

[15] Mori K, Hasegawa M, Nishida M, et al. Expression levels of thymidine phosphorylase and dihydropyrimidine dehydrogenase in various human tumor tissues. Int $\mathrm{J}$ Oncol 2000, 17: pp 33-38.

[16] Schüller J, Cassidy J, Dumont E, et al. Preferential activation of capecitabine in tumor following oral administration to colorectal cancer patients. Cancer Chemother Pharacol 2000, 45: pp 291-297.

[17] Mackean M, Planting A, Twelves C, et al. Phase I and pharmacologic study of intermittent twice-daily oral therapy with capecitabine in patients with advanced and/or metastatic cancer. J Clin Oncol 1998, 16: pp 2977-2985.

[18] Judson IR, Beale PJ, Trigo JM, et al. A human capecitabine excretion balance and pharmacokinetic study after administration of a single oral dose ${ }^{14} \mathrm{C}$-labelled drug. Invest New Drugs 1999, 17: pp 49-56.

[19] Cassidy J, Twelves C, Van Cutsem E, et al. First-line oral capecitabine therapy in metastatic colorectal cancer: a favorable safety profile compared with iv 5-fluorouracil (5-FU)/leucovorin. Ann Oncol 2002, 13: pp 566-575.
[20] Reigner B, Biesch K, Weidekamm E. Clinical pharmacokinetic of capecitabine. Clin Pharmacokinet 2001, 40: pp 85-104.

[21] Meredith PA. Potential concerns about generic substitution: bioequivalence versus therapeutic equivalence of different amlodipine salt forms. Curr Med Res Opin 2009, 25: pp 2179-2189.

[22] Chachad S, Purandare S, Malhotra G, Naidu R. Comparison of phamacikinetics and safety profiles of two capecitabine tablet formulations in patients with colon, colorectal or breast cancer. Cancer Chemother Pharmacol 2013; 71: 287-292.

[23] Duarte G, Babadopulos T, Shi L, et al. The use of healthy volunteers to evaluate bioequivalence of anti-neoplasic drugs: pilot studies with capecitabine. J Bioequiv Availab 2011; S1: $1-8$.

[24] Judson IR, Beale JP, Trigo JM, et al. A human capecitabine excretion balance and pharmacokinetic study after administration of a single oral dose of 14C-labelled drug. Invest New Drugs 1999, 17: pp 49-56.

[25] Reigner B, Blesch K, Weidekamm E. Clinical pharmacokinetic of capecitabine. Clin Pharmacokinet 2001, 40: pp 85-104.

[26] Reigner B, Verweij J, Dirix L, et al. Effect of food on the pharmacokinetics of capecitabine and its metabolites following oral administration in cancer patients. Clin Cancer Res 1998, 4: pp 941-948.

[27] Reichardt P, von Mickwitz G, Luck HJ, et al. Capecitabine: the new standard in metastatic breast cancer failing anthracyclineand taxane-containing chemotherapy? Mature results of a large multicenter phase II trial. Eur J Cancer 2001, 37 (Suppl 6): 191 (Abstract 699).

[28] Meredith PA. Potential concerns about generic substitution: bioequivalence versus therapeutic equivalence of different amlodipine. Curr Med Res Opin 2009, 25: pp 2179-2189.

[29] Johnston A, Asmar R, Dahlöf B, et al. Generic and therapeutic substitution: a viewpoint on achieving best practice in Europe. Br J Clin Pharnacol 2011, 72(5): pp 727-730. 